\title{
Generation of Electricity from Biological Source
}

\author{
Amey Kulkarni ${ }^{1}$, Amit Breed ${ }^{2 *}$ \\ ${ }^{1}$ Student, Department of Biotechnology, KET's V. G. Vaze College (Autonomous), Mumbai, India \\ ${ }^{2}$ Assistant Professor, Department of Biotechnology, KET's V. G. Vaze College (Autonomous), Mumbai, India \\ *Corresponding author: drasbreed@gmail.com
}

\begin{abstract}
Rapid consumption of renewable energy resources has led to development of an alternative source of energy. Fuel cell technology is a reliable and sustainable source of energy which was developed. Microbial fuel cell is a type which uses active microorganisms as catalysts for production of electricity. The microorganisms degrade the organic substrate to release protons and electrons which generate a potential difference across the cell. Our study focused on the generation of electricity from human urine using microbial fuel cell system. Specific bacteria were used as inoculum at anaerobic anode chamber and salt solution was supplied at aerobic cathode. The chambers were connected using salt bridge which would facilitate ion transfer. This made the system cost effective. The potential difference generated was measured using digital multi-meter.
\end{abstract}

Keywords: Aerobic cathode, Anaerobic anode, Cost effective, Microbial fuel cell, Micro-organisms, Potential difference, Salt bridge, Sustainable source.

\section{Introduction}

Sustainable energy production had been a task for over a decade. Due to rapid consumption of the available energy resources and the amount of pollution caused by them, there's a need for development of alternative source of energy. Many such efforts are taken in this field due to which we have several other alternative energy sources like solar energy, wind energy, hydroelectricity etc. One such effort lead to development of fuel cell technology. These fuel cells generate energy using high value metal catalyst (in traditional version). Fuel cell is of plethora advantages over other kinds of energy generators. FC is of plethora advantages over other kinds of energy generators, e.g. no emissions of environmental polluting gases (such as SOx, NOx, CO2 and CO), higher efficiency, no existence of mobile parts, as a result, lack of sonic pollution, and so forth [Rahimnejad M. et al. 2015].

One such type of fuel cell is microbial fuel cell [MFC]. MFC's are bio-electrochemical technology which converts organic waste to electricity [Potter MC, electrical effects accompanying the decomposition of organic compounds. Proc R SocB 1911;84;260;76]. The electrical current produced by bacteria was observed by Potter in 1911[Rahimnejad, M. et al., 2015]. Bio-mass is increasingly recognized as a green source of energy and waste streams are being regarded as the most optimum substrates for bioenergy production [Deval A. et al., 2013].

MFC's use active organisms as catalysts for generation of electricity. A standard MFC consists of 2 chambers, anode and cathode connected through a salt bridge which facilitate the transfer of electrons and protons. The electrons travel from anode to cathode through external load and cations diffuse through [Papaharalabos G. et al. 2015] salt bridge. The transfer of electrons across the cell leads to development of a potential difference between the two electrodes. This potential difference is measured using a digital multi-meter.

Microbial fuel cells (MFCs) have emerged in recent years as a promising yet challenging technology [Pant D. et al. 2010]. Over the last three decades, research in the field has focused on (i) improving the power density of individual units (ii) reducing the cost of each unit (iii) assembling units into stacks to reach exploitable power (iv) widening the range of potential fuels and (v) demonstrating the implementation of this biotechnology into practical applications [Walter X. A. et al. 2016]. The low output of the MFC had delayed this technology from reaching to its practical applications. Several MFC system have conceptually demonstrated to use variety of organics as substrates to generate electricity including glucose, acetate, sodium lactate, sucrose, propionate, butyrate, azo dye, chlorophenol and others [Huang J. et al. 2011]. The efficiency and economic viability of converting organic wastes to bioenergy depend on the characteristics and components of the waste material. The chemical nature of the waste material and the suitable bacteria used for the degradation of the same are major elements in the process of electricity production. These systems are also used for the degradation of the nitrates and sulphates and useful in wastewater treatment as they show effective reduction in chemical oxygen demand along with production of electricity.

Our study focused on the generation of electricity from Human urine as urine is normally just flushed down the toilet [You J. et al. 2016]. The human urine is composed of urea, uric acid, ammonia and several other constituents. These constituents serve as source for the growth and survival of the bacteria. The electroactive organism Pseudomonas aeruginosa breaks down the substrate and releases protons and electrons which travel through salt bridge and external circuit respectively. This flow of electrons and protons generated a potential difference across the circuit which was measured using a digital multi-meter. The maximum output voltage generated from the circuitry was $0.78 \mathrm{~V}$. The system was 
operated under ambient temperature conditions and the $\mathrm{pH}$ of the sample was measured at regular intervals.

MFC technology had been known to us from several years but its research mainly focused on widening the range of potential fuels and its practical applications. The limitations of the MFC include low power output as it is a biological process and high cost. Thus, the aim of the research was to develop a cost effective and efficient MFC model which would generate electricity from urine and practically apply it at the urinals to make them self-sufficient. The materials used heavily reduced the MFC cost. Further optimization of the process needs to be done to increase the output of the cell.

\section{Materials and Methods}

\section{A. MFC Construction and Design}

The model used was constructed of plastic bottles, and a salt bridge instead of a proton exchange membrane as the mediator for transfer of electrons.

The microbial fuel cell consisted of 2 chambers anode and cathode. The chambers were made up of plastic containers having capacity of $1 \mathrm{~L}$ each. The anode container was closed completely so as to maintain anaerobic conditions. The cathode container was exposed to air. Both the chambers were connected using KCl-Agar salt bridge. The graphite rods were used as electrodes and were placed in both the chambers i.e. anode and cathode.

\section{B. Sample Collection}

\section{Urine:}

In this paper, focus was made on Human urine as the sample. The urine sample was collected from 2 healthy individuals.

The anaerobic anode chamber was supplied with $500 \mathrm{ml}$ of fresh urine sample and the cathode with equal amount of saturated salt solution as an electrolyte.

\section{Culture preparation}

Pseudomonas aeruginosa is a gram-negative organism which grows on nutrient agar. Thus, a loopful of culture was inoculated on nutrient agar slants and was kept for incubation at $37^{\circ} \mathrm{C}$ for 48 hours. After 48 hours the culture was transferred to a saline solution. This was used as the inoculum in the urine sample.

\section{Operation of $M F C$ 's}

The MFC's were fed with the urine and were connected to a digital multi-meter [unity A830L] for measuring the output voltage of the cell.

The MFC's were kept at optimum room temperature and were monitored for over a period of 72 hours. The $\mathrm{pH}$ of the sample was measured every 06 hours.

\section{Results and Discussion}

Table 1

\begin{tabular}{|l|l|l|}
\hline \multicolumn{3}{|c|}{ Parameters of the MFC } \\
\hline PH & \multicolumn{1}{|c|}{ Initial } & Final \\
\hline Temperature range & 5.0 & 10.0 \\
\hline
\end{tabular}

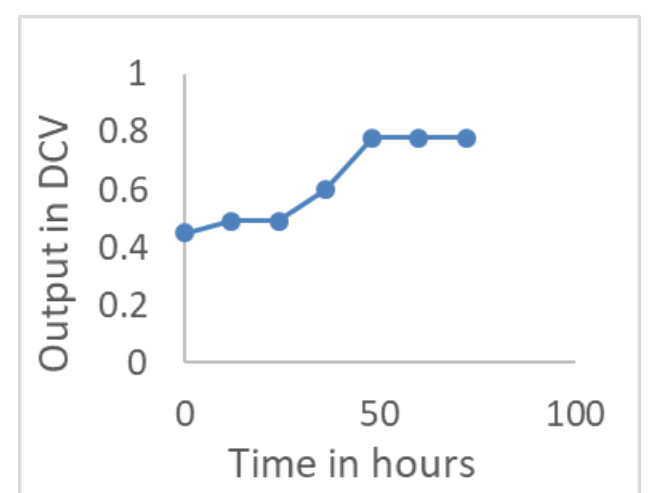

Fig. 1. Graph representing the average output voltage w.r.t time

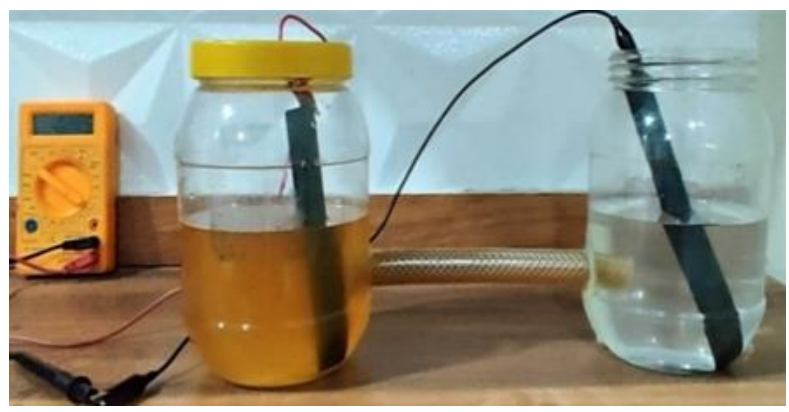

Fig. 2. Two chambered MFC

It was observed that the output voltage increased up to 48 hours (figure 1). After 48 hours the stabilization in the output voltage was observed.

The inoculation of bacteria to the human urine led to a steady increase in the output voltage of the cell which was observed for 48 hours. The urine sample having 1:1 ratio with salt water at cathode produced a maximum voltage of $0.78 \mathrm{~V}$. The initial and the final $\mathrm{pH}$ of the urine sample was measured to be 5.0 and 10.0 respectively. Decrease in the voltage was observed after 48 hours which shows that fresh urine needs to be circulated.

\section{B. Factors affecting the output}

There were several factors influencing the electricity generation process. Controlling of these factors would even lead to a higher output. The factors were $\mathrm{pH}$, growth of the micro-organisms, surface area of the electrodes, availability of electron acceptor at cathode and temperature. Temperature plays an important role in the growth and multiplication rate of the organism thus it is an important factor to be controlled. The system functions to its fullest at the temperature range of 28 $35^{\circ} \mathrm{C}$ with an alkaline $\mathrm{pH}$ range. The surface area of electrodes also plays an important role as larger the surface area greater the flow of electrons towards it and thus greater output.

\section{A. Observations}

Urine MFC: 


\section{Conclusion}

The study demonstrated the feasibility of bioelectricity generation from human urine using a low-cost MFC. Several factors including $\mathrm{pH}$, temperature, played an important role in the electricity generation process. This system shifted the $\mathrm{pH}$ of the samples from acidic to alkaline and generated electricity. Thus, MFC's are promising technology in the development of sustainable energy.

\section{Future scope}

The further research would focus on maximizing the output of the system and simultaneously monitoring the process parameters such as temperature, $\mathrm{pH}$, surface area of the electrodes, growth of the micro-organisms which affect the output. It would also include the standardization of the process.

\section{Acknowledgement}

We would like to thank the Department of Biotechnology (DBT) star scheme for funding the project. We sincerely express our gratitude towards the Department of Biotechnology of V. G. Vaze College for its support and allowing us to present the project at the conference.

\section{Conflict of Interest}

The project was selected in order to develop an alternative energy source using the MFC. The human urine was selected as the substrate for electricity generation as it would have potential large-scale applications at the public urinals in order to make them self-powered.

\section{References}

[1] Deval, A., Dikshit, Anil kumar. (2013). Construction, working and standardisation of MFC. APCBEE Procedia, 5: 59-63.

[2] Pant, D., Bogaert, G.V., Diels, L., Vanbroekhoven, K. (2010). A review of the substrates used in microbial fuel cells (MFCs) for sustainable energy production. Bioresource Technology, 101(6): 1533-1543.

[3] Papaharalabos, G., Greenman, J., Melhuish, C., Ieropoulos, I. (2015). Novel small scale MFC design for increased electricity generation. International journal of hydrogen energy, 40(11): 4263-4268.

[4] Huang, J., Yang, P., Guo, Y., Zhang, K. (2011). Electricity generation during wastewater treatment: An approach using an AFB-MFC for alcohol distillery wastewater. Desalination, 276: 373-378.

[5] You, J., Greenman, J., Melhuish, C., Ieropoulos, I. (2016). Electricity generation and struvite recovery from human urine using microbial fuel cells. Journal of Chemical technology and Biotechnology, 91(3): 647-654.

[6] Rahimnejad, M., Ahami, A., Darvari, S., Zirepour, A., Sang-Eun Oh. (2015). Microbial fuel cell as new technology for bioelectricity generation: A review. Alexandria engineering journal 54(3): 745-756.

[7] Walter, X.A., Stinchcombe, A., Greenman, J., Ieropoulos, I. (2016). Urine transduction to usable energy. Applied energy, 192: 575-581. 